\title{
Nutritional influences on mood and cognitive performance: the menstrual cycle, caffeine and dieting
}

\author{
BY PETER J. ROGERS, STEPHEN EDWARDS, MICHAEL W. \\ GREEN AND PAULINE JAS \\ Consumer Sciences Department, AFRC Institute of Food Research, Reading Laboratory, \\ Earley Gate, Whiteknights Road, Reading RG6 2EF
}

It is widely believed that there exists a strong relationship between eating and mood, and that diet and particular dietary constituents can have important influences on behaviour, including alertness and mental (cognitive) performance. An obvious example is caffeine, thought to be the most frequently consumed drug in the world (Gilbert, 1984), which is often consumed with the specific intention to alter mood; for instance strong coffee consumed at breakfast for its alerting effect. Longer-term effects of diet on behaviour are more difficult to study, because long-term dietary manipulations are difficult to impose and in correlational studies differences in diet are always confounded with other variables. Nonetheless, the recent concern over possible adverse behavioural consequences of low-fat diets (e.g. Engleberg, 1992) confirms the need for systematic studies of diet-behaviour relationships.

The main aim of the present article is to examine some of the mechanisms underlying nutritional effects on mood and cognitive performance, and also to indicate how these effects can feed back to influence food preference and liking. This second issue has been largely neglected in the research carried out in this field, even though it forms the basis of at least one prominent theory linking mood effects and food choice (Wurtman \& Wurtman, 1989). Both issues will be illustrated by reference to the subject-matter set out in the title, namely the effects of the menstrual cycle, caffeine and dieting.

\section{THE MENSTRUAL CYCLE, MOOD AND EATING}

Changes in mood, food intake and food choice associated with the menstrual cycle have been documented in numerous anecdotal reports and a variety of experimental studies (for references, see p. 344). Moreover, it has been suggested that altered nutrient intakes may act to ameliorate the negative shift in mood which is experienced during the premenstrual phase by at least a significant proportion of women (Wurtman \& Wurtman, 1989). The menstrual cycle, therefore, would appear to provide a valuable model for examining the interrelationships between mood and eating. Currently, however, there is little consensus in this field. This may be due partly to the complexity of the psychosocial and biological mechanisms which probably underlie the cyclic changes in mood and behaviour, and partly to methodological difficulties concerning, for example, mood measurement and the lack of accurate determination of menstrual cycle phase. Some authors have concluded that menstrual cycle effects on mood and eating occur largely independently (e.g. Cohen et al. 1987; Bancroft et al. 1988). These studies, however, relied on daily ratings of mood and behaviour, and consequently may have lacked sufficient resolution to detect the subtle mood changes which are associated with individual eating episodes (de Castro, 1987; Hill et al. 1991). 
Perhaps the most consistent finding is that energy intake is relatively increased during the premenstrual (luteal) phase, although the exact timing and size of this increase differs considerably between studies (e.g. Dalvit-McPhillips, 1983; Manocha et al. 1986; Lissner et al. 1988; Tarasuk \& Beaton, 1991). In their careful analysis Lissner et al. (1988) found that energy intake was highest in the mid-luteal phase and lowest immediately before and during menstruation. There was also evidence of a second, smaller peak in intake during the postmenstrual (follicular) phase.

Wurtman \& Wurtman's (1989) argument is based on evidence of a selective increase in the consumption of high-carbohydrate foods during the premenstrual phase (e.g. Dalvit-McPhillips, 1983). The hypothesis specifically applies to premenstrual syndrome (PMS), and also to conditions such as seasonal affective disorder, where mood fluctuations can be particularly severe. The change in nutrient intake is supposed to alter uptake of tryptophan into the brain, brain serotonergic activity, and in turn ameliorate the disturbances of mood. Furthermore, it is claimed that the increase in carbohydrate intake is driven by an increase in preference (liking) for high-carbohydrate foods which has been termed 'carbohydrate craving'. This is of considerable interest because it implicates effects of food on mood as a factor influencing food acceptance. It is possible that this occurs through a conditioning process similar to that which has been investigated extensively in studies examining the development of learned aversions for foods producing sickness and the acquisition of preferences for foods conferring nutritional benefit. Such learned preferences and aversions appear to be characterized by alterations in the pleasantness or liking of the foods' taste, flavour, texture etc. (Rogers \& Blundell, 1990). At present, however, there is little direct evidence as to whether or not sensory preferences can be conditioned by the effects of food on mood (see also the effects of caffeine, p. 346).

More serious objections to Wurtman \& Wurtman's (1989) proposals concern the precise nature of the changes in nutrient intakes and how these changes can be expected to influence the functional activity of brain serotonergic systems. For example, in humans the likely effect of carbohydrate ingestion on brain uptake of tryptophan (the amino acid precursor of serotonin) is markedly reduced if only a small amount of protein is included in the meal or snack (Teff et al. 1989; see also Leathwood, 1987; Young, 1991). Also it would seem that the term 'carbohydrate craving' is largely a misnomer, since what have been termed carbohydrate snacks are in fact high in both fat and carbohydrate (Drewnowski, 1991). Thus, in one recent study of PMS, premenstrual increases in energy intake were found to be due predominantly to an $18 \%$ increase in fat intake, accompanied by increases in preference for high-fat as well as high-carbohydrate foods (A. J. Hill and J. E. Blundell, unpublished results). Other studies, involving women not diagnosed as suffering from PMS, indicate that chocolate is a frequently craved food during the premenstrual phase (Tomelleri \& Grunewald, 1987; Rozin et al. 1991) and again that increases in fat intake account for the premenstrual increases in energy intake (Tarasuk \& Beaton, 1991).

From the previous discussion it is apparent that the relationships between food preference, mood and the menstrual cycle are not well understood. Ovarian hormones may exert a direct action on appetite and food intake, but it is unlikely that this alone can explain either the cyclic fluctuations in total energy intake (Lissner et al. 1983) or the more variable changes in food choice. Other explanations include the possibility that these changes function to alleviate negative mood states, not via a nutritional mechanism 
(Wurtman \& Wurtman, 1989), but by the powerful hedonic effects accompanying the consumption of certain foods. In particular it would appear that chocolate is frequently eaten for its positive effect on mood, to increase pleasure, to relieve boredom, to relieve anxiety, or as a source of comfort, a capacity which is probably related to its sensory properties and the role it fulfils in our food culture (Hill et al. 1991; Rogers, 1992).

Negative mood might also be implicated as a factor precipitating a loss of motivation to limit food intake and avoid certain 'forbidden' foods viewed as fattening or 'dietbreaking'. In laboratory studies, induction of negative mood has been reported to trigger overeating in dieters and highly-restrained eaters (Herman \& Polivy, 1975; Ruderman, 1985). Furthermore, since dieting itself is a source of stress (Herman \& Polivy, 1991), it is possible that premenstrual changes in mood are exacerbated in dieters. As far as we are aware, however, there has been only one study which has attempted to examine the interaction between menstrual cycle effects and dietary restraint (Cohen et al. 1987). Mood was found to be less positive, and food consumption and food cravings greater during the premenstrual phase, but dietary restraint was related only to craving. Surprisingly, the 'dieters' reported less food craving than the 'non-dieters'.

Still another issue that needs to be addressed in relation to menstrual cycle effects on mood and behaviour concerns the role of individual expectations and attributions. Ussher (1989), for example, has pointed out that there is great variability in mood changes associated with the premenstrual phase, with some women reporting predominantly negative moods, including tension and anxiety, irritability, and depression, but other women reporting improved mood, in particular, feelings of increased 'energy'. Ussher (1989) argues that underlying these different experiences is a premenstrual increase in arousal or activation (see also Thayer, 1989), which can be interpreted by individual women in different ways. Furthermore, it appears that negative mood changes are more likely to be attributed to biological factors, whereas positive moods are likely to be attributed to personality or situational factors (Ussher, 1989). Similarly, the premenstrual state might also be used as an attribution (personal explanation or 'excuse') for the relaxation of dietary restraint. This underlines the complexity of menstrual cycle effects and the need to take into account the potentially powerful influence of individual expectations, especially in research where the participating women are aware that the menstrual cycle is the subject of study.

\section{CAFFEINE}

Caffeine has a variety of physiological actions, which are probably mediated by the blockade of adenosine receptors (James, 1991). Although the direct central nervous system (CNS) effects of caffeine are not well established, the widely accepted description of caffeine as a CNS stimulant is consistent with many of the behavioural effects of this substance, which include feelings of increased alertness, a quickening of reaction time, and enhanced vigilance performance, but decreased hand steadiness (Lieberman et al. 1987; James, 1991). An important, but neglected, question concerns how these effects on mood and behaviour are related to the preference, liking and consumption of caffeinecontaining beverages. One difficulty here is that many of the laboratory studies examining the effects of caffeine have used acute doses higher than those that are normally obtained from drinking, for example, tea and coffee. Nonetheless, definite behavioural effects are evident at relatively low doses (Lieberman et al. 1987), and there 
is good evidence that humans can discriminate caffeine from placebo at very low doses (10-56 mg; Griffiths et al. 1990).

As indicated previously, expectation of increased alertness and improved concentration would appear to be one reason for choosing to drink coffee. Equally, however, strong coffee may be avoided in the late evening because of the expectation that caffeine ingestion can lead to difficulty in getting to sleep and poorer quality of sleep. In other words caffeine-containing beverages are perceived as having both positive and negative consequences. This is confirmed by several studies which have examined the reinforcing properties of caffeine. These studies, which have been reviewed recently by James (1991), show that caffeine can serve as a positive reinforcer and also as a 'punisher' depending on a variety of factors, including dose, background level of caffeine consumption, contextual variables and individual subject differences. For example, Griffiths \& Woodson (1988) used a double-blind procedure in which subjects chose between different coloured capsules containing either placebo or caffeine (four doses between 100 and $600 \mathrm{mg}$ tested). Subjects made their choice between caffeine and placebo after overnight abstinence from caffeine. Five of the twelve subjects showed a consistent preference for caffeine at one or more of the four doses. Using a similar method, Stern et al. (1989) found overall that the choice of a $100 \mathrm{mg}$ dose of caffeine did not differ from chance, whereas a higher dose $(300 \mathrm{mg})$ was chosen on significantly fewer occasions than expected by chance. However, when the subjects were divided into caffeine 'choosers' and 'non-choosers' a clear relationship emerged between drug choice and subjective effects, with non-choosers reporting primarily aversive effects associated with caffeine consumption (increased anxiety and dysphoria) and choosers reporting positive mood effects such as increase in 'vigour'.

These reinforcing effects of caffeine that are seen in some individuals could presumably provide the basis for the development of preferences for caffeine-containing beverages through a flavour-'drug' conditioning process, similar in principle to flavourenergy conditioning (Elizalde \& Sclafani, 1988; Zellner, 1991; see also p. 344). We have attempted to demonstrate such an effect by pairing caffeine ingestion with the consumption of novel-flavoured fruit juices (P. J. Rogers, S. Edwards and D. J. Mela, unpublished results). However, all the measures of preference indicated that there was an increase in liking for the non-caffeine-paired flavour during the course of the experiment, but that there was little or no change in liking for the caffeine-paired flavour (some of the results are shown in Table 1). This suggests that, if anything, caffeine had a negative influence, preventing the increase in preference associated with increasing familiarity with the flavours. A more comprehensive study with measures of mood and two dose levels of caffeine included is currently in progress.

This method may well prove to be of considerable value in examining the relationships between the presence of potentially 'psychoactive' constituents and the acquisition of preferences for certain foods and beverages. On the current evidence, however, it must be concluded that there is little direct support for the idea that caffeine plays a major role in the development of the liking for caffeine-containing beverages. This does not mean that the effects of caffeine are unimportant. Clearly, caffeine does influence mood and behaviour and, furthermore, there are well-known symptoms of withdrawal from caffeine, which include headache, drowsiness and fatigue (Griffiths et al. 1986; Hughes et al. 1991). Avoidance of these negative effects of withdrawal is likely to contribute to at least the maintenance of preferences for caffeine-containing beverages among frequent users. 
Table 1. Pre-and post-conditioning preferences $\dagger$ for fruit-juice drinks paired with or without the ingestion of caffeine $(75 \mathrm{mg}$ )

(Mean values with their standard errors)

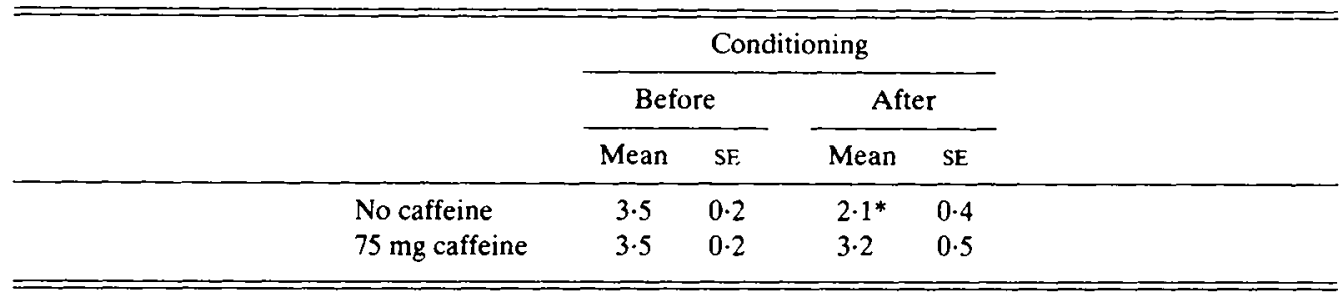

Mean value was significantly different from that before conditioning: ${ }^{*} P<0.02$.

$\uparrow$ Rank order preference; the two drinks consumed during conditioning were assessed against a further four drinks ( 1 most preferred, 6 least preferred).

Other factors which may be involved have been discussed by Zellner (1991) in relation to the liking for coffee. She notes that coffee is initially strongly disliked since it contains bitter constituents (including caffeine), and bitter substances are innately rejected by humans (Steiner, 1987). This may be circumvented by the addition of sweeteners and milk or cream which can improve the sensory appeal of the beverage and provide the opportunity for flavour-flavour conditioning (coffee flavours paired with strongly liked flavours) and flavour-energy conditioning (coffee flavours paired with sugar and fat energy) to occur. In addition, social influences will be important in shaping early exposure to coffee and other caffeine-containing beverages. As a result, liking and the habit of consuming these beverages may develop through modelling others' behaviour, and through an 'evaluative' conditioning process whereby liking may be increased as a consequence of pairing the drinking of, for example, coffee with a positive social situation (e.g. presence of family or friends) and positive mood.

\section{DIET, DIETARY RESTRAINT AND DIETING}

Another example of the relationship between nutrition and behaviour where multiple mechanisms are likely to be involved concerns the effects of dieting and dietary restraint. Dieting is a major preoccupation of consumers in many economically developed countries, and occurs in children as well as adults (Hill et al. 1992). There has been a large amount of research on, for example, 'restrained eating' and concern about body shape, and their influences on food choice and intake control (discussed briefly on p. 345 in relation to menstrual cycle effects). The metabolic and nutritional consequences of food restriction (Garrow, 1988) have also been studied extensively. However, while some work has examined mood changes during different dieting regimens (e.g. Rosen et al. 1985), the possible effects of dieting on cognitive performance have been almost completely ignored.

The Minnesota study of semi-starvation (Keys et al. 1950) showed that long-term food restriction results in lethargy, tiredness, depression and greatly decreased feelings of 'energy', which clearly would be expected to be associated with impaired cognitive performance. However, although the subjects complained of a number of changes in their intellectual functioning, including an inability to concentrate, impaired judgement 
Table 2. Different types of dieting' behaviour

\author{
Missing a meal \\ 'Healthy eating' (e.g. low- or reduced-fat diet) \\ Dieting to maintain weight \\ Dieting to lose weight \\ Intermittent food restriction or starvation (e.g. bulimia nervosa) \\ Chronic, severe semi-starvation (e.g. anorexia nervosa)
}

and poorer memory, this was not confirmed by the results of a battery of 'objective' tests of performance. Any effects observed were only marginal. Perhaps the subjects' estimation of their intellectual capacity was influenced by expectations arising from the emotional and physical effects of starvation. It is also possible, however, that the performance-testing protocol or the tests themselves were relatively insensitive. One problem is that there was no adequate control group to assess the effects of repeated testing. Consequently, improved performance due to practice may have compensated for deterioration in performance due to food restriction.

Table 2 lists various forms of 'dieting' behaviour which involve different degrees of energy restriction and changes in patterns of eating and nutrient intake. The Minnesota study represents an extreme example of 'dieting', although of course if the goal is to lose a significant amount of body fat, the dieter must sustain negative energy balance for some considerable period of time. The avoidance of weight gain (i.e. maintenance of current weight) does not require the 'dieter' to undereat but, nonetheless, for some individuals the effort of maintaining dietary restraint or the effects of alternating between dietary restraint and diet breaking may be sufficiently stressful to adversely affect mood and cognitive performance (Herman \& Polivy, 1991). On the other hand, successful attainment of weight-loss targets during dieting may well lead to an improvement in mood, body esteem and self-regard (Garrow, 1991).

We have begun to examine some of these issues in a study in which women undergraduates were assessed for their current dieting behaviour and their concerns about eating, body-weight and body shape (Rogers \& Green, 1992). They were also tested on a demanding, rapid information processing task. In this procedure, known as the Bakan task, a continuous stream of single digits was presented on a computer visual display unit at the rate of 100 digits per min. The subjects were required to press a response button whenever they detected a sequence of three odd or three even digits. A total of forty such targets were presented over 5 min each separated by a minimum of five and a maximum of thirty digits.

Thirteen of the fifty-five volunteers reported that they were currently dieting to lose weight. These subjects were compared with the remaining subjects divided into low-to-medium-restraint and high-restraint groups on the basis of their scores on the dietary restraint factor of the English version of the Dutch Eating Behaviour Questionnaire (van Strien et al. 1986). The dietary restraint scale from this questionnaire is a measure of concerns about eating in relation to body-weight. Some of the results are shown in Table 3. The currently-dieting subjects did not differ from the high-restraint subjects on dietary restraint or body mass index, although the results for the body-shape 
Table 3. Body size, body shape concerns and cognitive performance as a function of dietary restraint and current dieting behaviour

(Mean values with their standard errors)

\begin{tabular}{|c|c|c|c|c|c|c|}
\hline \multirow[t]{2}{*}{$n \ldots$} & \multicolumn{2}{|c|}{$\begin{array}{l}\text { Low-to-medium } \\
\text { restraint } \\
27\end{array}$} & \multicolumn{2}{|c|}{$\begin{array}{c}\text { High restraint } \\
15\end{array}$} & \multicolumn{2}{|c|}{$\begin{array}{c}\text { Currently dieting } \\
\text { to lose weight } \\
13\end{array}$} \\
\hline & Mean & SE & Mean & $\mathrm{SE}$ & Mean & $\mathrm{SE}$ \\
\hline Dietary restraint (maximum 5) & $2 \cdot 2^{\mathrm{a}}$ & $0 \cdot 1$ & $3 \cdot 6^{b}$ & $0 \cdot 1$ & $3 \cdot 7^{b}$ & $0 \cdot 2$ \\
\hline Body mass index $\left(\mathrm{kg} / \mathrm{m}^{2}\right)$ & $20 \cdot 3^{a}$ & 0.3 & $22 \cdot 1^{b}$ & 0.4 & $22 \cdot 7^{b}$ & 0.7 \\
\hline Body shape questionnaire & $72^{a}$ & 4 & $84^{a}$ & 5 & $115^{\mathrm{b}}$ & 5 \\
\hline Bakan task ( $\%$ targets detected) & 48 & 2 & 49 & 4 & $40 *$ & 5 \\
\hline
\end{tabular}

a,b Mean values with unlike superscript letters were significantly different $(P<0 \cdot 01)$.

Mean value was significantly different from that for non-dieters (low-to-medium-restraint and high-restraint groups combined; one-tailed): ${ }^{*} P=0-039$.

questionnaire (Cooper et al. 1987) indicated greater dissatisfaction with body shape and weight among the dieters. The dieters also tended to perform less well than the non-dieters on the cognitive task. This last result was confirmed and extended in a second study (M. W. Green and P. J. Rogers, unpublished results) in which current dieters were found to be relatively impaired on rapid information processing (i.e. the same Bakan task), simple reaction time and immediate memory. Furthermore, performance was poorest in subjects who had been on the current diet the longest and had lost the most weight, while the group of highly-restrained but non-dieting subjects performed at a level intermediate between the low-to-medium restrainers and the current dieters. Heart rate immediately before and after testing was lowest in the current dieters with high weight loss, perhaps implicating lower arousal or alertness in these subjects as a factor mediating the poorer cognitive performance. Lowered heart rate is typical of a chronic state of undernutrition (Keys et al. 1950).

The previously described results indicate substantial impairment of cognitive performance with dieting. This could be due to a variety of factors, including temperamental differences between dieters and non-dieters. The effects, however, appeared to be related more closely to the degree of weight loss than to concern about eating and body-weight. This would implicate the severity or duration of energy restriction, or both, as a major factor contributing to the poorer cognitive efficiency of the dieters (Laessle et al. 1990), although differences in eating pattern and nutrient intake may also play an important role. In order to dissociate these different influences it will be necessary to carry out controlled, prospective studies of the effects of diet and dieting on behaviour.

\section{REFERENCES}

Bancroft, J., Cook, A. \& Williamson, L. (1988). Food craving, mood and the menstrual cycle. Psychological Medicine 18, 855-860.

Cohen, I. T., Sherwin, B. B. \& Fleming, A. S. (1987). Food cravings, mood, and the menstrual cycle. Hormones and Behaviour 21, 457-470.

Cooper, P. J., Taylor, M. J., Cooper, Z. \& Fairburn, C. G. (1987). The development and validation of the body shape questionnaire. International Journal of Eating Disorders 6, 485-494. 
Dalvit-McPhillips, S. P. (1983). The effect of the human menstrual cycle on nutrient intake. Physiology and Behaviour 31, 209-212.

de Castro, J. M. (1987). Macronutrient relationships with meal patterns and mood in spontaneous human feeding behaviour. Physiology and Behaviour 39, 561-569.

Drewnowski, A. (1991). Fat and sugar: Sensory and hedonic aspects of sweet, high-fat foods. In Chemical Senses. Vol. 4, Appetite and Nutrition, pp. 69-83 [M. I. Friedman, M. G. Tordotf and M. R. Kare, editors]. New York: Marcel Dekker.

Elizalde, G. \& Sclafani, A. (1988). Starch-based conditioned preferences in rats: influence of taste, calories and CS-US delay. Appetite 11, 179-200.

Engleberg, H. (1992). Low serum cholesterol and suicide. Lancet 339, 727-729.

Garrow, J. S. (1988). Obesity and Related Diseases. London: Churchill Livingstone.

Garrow, J. S. (1991). The safety of dieting. Proceedings of the Nutrition Society 50, 493-499.

Gilbert, R. M. (1984). Caffeine consumption. In The Methylxanthine Beverages and Food, pp. 185-213 [G. A. Spiller, editor]. New York: Alan R. Liss.

Griffiths, R. R., Bigelow, G. E. \& Liebson, I. A. (1986). Human coffee drinking: Reinforcing and physical dependence producing effects of caffeine. Journal of Pharmacology and Experimental Therapeutics 239, $416-425$.

Griffiths, R. R., Evans, S. M., Heishman, S. J., Preston, K. L., Sannerud, C. A., Wolf, B. \& Woodson, P. P. (1990). Low-dose caffeine discrimination in humans. Journal of Pharmacology and Experimental Therapeutics 252, 970-978.

Griffiths, R. R. \& Woodson, P. P. (1988). Reinforcing effects of caffeine in humans. Journal of Pharmacology' and Experimental Therapeutics 246, 21-29.

Herman, C. P. \& Polivy, J. (1975). Anxiety, restraint and eating behaviour. Journal of Abnormal Psychology $85,666-672$.

Herman, P. \& Polivy, J. (1991). Fat is a psychological issue. New Scientist 1795, 41-45.

Hill, A. J., Oliver, S. \& Rogers, P. J. (1992). Eating in the adult world: The rise of dieting in childhood and adolescence. British Journal of Clinical Psychology 31, 95-105.

Hill, A. J., Weaver, C. F. L. \& Blundell, J. E. (1991). Food craving, dietary restraint and mood. Appetite 17, 187-197.

Hughes, J. R., Higgins, S. T., Bickel, W. K., Hunt, W. K., Fenwick, J. W., Gulliver, S. B. \& Mireault, G. C. (1991). Caffeine self-administration, withdrawal, and adverse effects among coffee drinkers. Archives of General Psychiatry 48, 611-617.

James, J. E. (1991). Caffeine and Health. London: Academic Press.

Keys, A., Brozek, J., Henschel, A., Mickelsen, O. \& Taylor, H. F. (1950). The Biology of Human Starvation, vols. 1 and 2. Minneapolis: University of Minnesota Press.

Laessle, R. G., Bossert, S., Hank, G., Hahlweg, K. \& Pirke, K. M. (1990). Cognitive performance in patients with bulimia nervosa: Relationship to intermittent starvation. Biological Psychiatry 27, 549-551.

Leathwood, P. D. (1987). Tryptophan availability and serotonin synthesis. Proceedings of the Nutrition Society 46, 143-156.

Lieberman, H. R., Wurtman, R. J., Emde, G. C., Roberts, C. \& Coviella, I. L. G. (1987). The effects of low doses of caffeine on human performance and mood. Psychopharmacology 92, 308-312.

Lissner, L., Stevens, J., Levitsky, D. A., Rasmussen, K. M. \& Strupp, B. J. (1988). Variation in energy intake during the menstrual cycle: implications for food-intake research. American Journal of Clinical Nutrition 48, 956-962.

Manocha, S., Choudhuri, G. \& Tandon, B. N. (1986). A study of dietary intake in pre- and post-menstrual periods. Human Nutrition: Applied Nutrition 40A, 213-216.

Rogers, P. J. (1992). Mechanisms of moreishness and food craving. In Pleasure, Politics and the Reality, [D. M. Warburton, editor] (In the Press).

Rogers, P. J. \& Blundell, J. E. (1990). Psychobiological bases of food choice. British Nutrition Foundation Nutrition Bulletin 15, Suppl. 1, 31-40.

Rogers, P. J. \& Green, M. W. (1992). Dieting, dietary restraint and cognitive performance. British Journal of Clinical Psychology (In the Press).

Rosen, J. C., Gross, J., Loew, D. \& Sims, E. A. H. (1985). Mood and appetite during minimal-carbohydrate and carbohydrate-supplemented hypocaloric diets. American Joumal of Clinical Nutrition 42, 371-379.

Rozin, P., Levine, E. \& Stoess, C. (1991). Chocolate craving and liking. Appetite 17, 199-212.

Ruderman, A. J. (1985). Dysphoric mood and overeating: A test of restraint theory's disinhibition hypothesis. Journal of Abnormal Psychology 94, 78-85. 
Steiner, J. E. (1987). What the neonate can tell us about umami. In Umami: A Basic Taste, pp. $97-123$ [Y. Kawamura and M. R. Kare, editors]. New York: Marcel Dekker.

Stern, K. N., Chait, L. D. \& Johanson, C. E. (1989). Reinforcing and subjective effects of caffeine in normal human volunteers. Psychopharmacology 98, 81-88.

Tarasuk, V. \& Beaton, G. H. (1991). Menstrual-cycle patterns in energy and macronutrient intake. American Journal of Clinical Nutrition 53, 442-447.

Teff, K. L., Young, S. N. \& Blundell, J. E. (1989). The effect of protein or carbohydrate breakfasts on subsequent plasma amino acid levels, satiety and nutrient selection in normal males. Pharmacology, Biochemistry and Behaviour 34, 829-837.

Thayer, R. E. (1989). The Biopsychology of Mood and Arousal. New York: Oxford University Press.

Tomelleri, R. \& Grunewald, K. K. (1987). Menstrual cycle and food cravings in young college women. Journal of the American Dietetic Association 87, 311-315.

Ussher, J. M. (1989). The Psychology of the Female Body. London: Routledge.

van Stein, T., Frijters, J. E. R., Bergers, G. P. A. \& Defares, P. B. (1986). The Dutch Eating Behaviour Questionnaire (DEBQ) for assessment of restrained, emotional, and external eating behaviour. International Journal of Eating Disorders 5, 295-315.

Wurtman, R. J. \& Wurtman, J. J. (1989). Carbohydrates and depression, Scientific American 260, 50-57.

Young, S. N. (1991). Some effects of dietary components (amino acids, carbohydrate, folic acid) on brain serotonin synthesis, mood and behaviour. Canadian Journal of Physiology and Pharmacology 69, 893-903.

Zellner, D. A. (1991). How foods get to be liked: some general mechanisms and some special cases. The Hedonics of Taste, pp. 199-217 [R. C. Bolles, editor]. Hillsdale, N.J.: Erlbaum, Lawrence, Associates, Inc. 